Pesq. Vet. Bras. 37(5):521-525, maio 2017 DOI: 10.1590/S0100-736X2017000500016

\title{
Efeitos da corticoterapia materna nos valores hemogasométricos de cordeiros nascidos a termo e prematuros ${ }^{1}$
}

\author{
Francisco Leydson Formiga Feitosa ${ }^{2 *}$, Larissa Gabriela Ávila ${ }^{3}$, Fernanda Bovino ${ }^{4}$, \\ Guilherme Gonçalves Fabretti Santos ${ }^{4}$, Luiz Cláudio Nogueira Mendes², Juliana \\ Regina Peiró ${ }^{2}$ e Sílvia Helena Venturolli Perri ${ }^{5}$
}

\begin{abstract}
Feitosa F.L.F., Ávila L.G., Bovino F., Santos G.G.F., Mendes L.C.N., Peiró J.R. \& Perri S.H.V. 2017. [Effects of maternal blood gas values corticotherapy of Lambs born at term and premature.] Efeitos da corticoterapia materna nos valores hemogasométricos de cordeiros nascidos a termo e prematuros. Pesquisa Veterinária Brasileira 37(5):521-525. Departamento de Clínica, Cirurgia e Reprodução Animal, Faculdade de Medicina Veterinária de Araçatuba, Universidade Estadual Paulista “Júlio de Mesquita Filho", Rua Clóvis Pestana 793, Jardim Dona Amélia, Araçatuba, SP 16050-680, Brazil. E-mail: leydsonf@fmva.unesp.br

The aim of the study was to evaluate blood gas parameters of full-term and premature lambs from birth to 48 hours of life. Four experimental groups were formed: NDG (normal delivery group - lambs vaginally delivered, $n=15$, average of 146-day gestation); NDEXG (normal delivery with dexamethasone group - lambs vaginally delivered whose mothers received $16 \mathrm{mg}$ of dexamethasone at 141 days of gestation, $n=8$, average of 143-day gestation); PRE (premature lambs born by cesarean section at 138 days of gestation, $n=10$ ) and PREDEX (premature lambs born by cesarean section at 138 days gestation, whose mothers received $16 \mathrm{mg}$ of dexamethasone two days before, $n=9$ ). Immediately after birth, lambs from all groups showed respiratory acidosis (low $\mathrm{pH}$ and high $\mathrm{pCO}_{2}$ ), most obviously in premature animals. The concentration of $\mathrm{HCO}_{3}{ }^{-}$was lower between 15 and 60 minutes of life, especially in PRE and PREDEX groups with subsequent increase in M24h. The values of base excess were lower in premature lambs, which showed abdominal breathing, severe dyspnea and lots of lung fluid. The stabilization of acid-base balance occurred in all animals during the first 24 hours of life. Dexamethasone had a positive effect on the clinical condition of the premature lambs, resulting in adequate ventilation and tissue perfusion, which guaranteed higher survival rate.

INDEX TERMS: Blood gas values, corticotherapy, hemogasometry, lambs, acid-base balance, blood gas analysis, prematurity, dexamethasone.
\end{abstract}

RESUMO.- 0 objetivo do estudo foi avaliar as variáveis hemogasométricas de cordeiros nascidos a termo e prematuros do nascimento às 48 horas de vida. Foram constituídos

\footnotetext{
${ }^{1}$ Recebido em 29 de julho de 2016.

Aceito para publicação em 7 de novembro de 2016.

${ }^{2}$ Departamento de Clínica, Cirurgia e Reprodução Animal, Faculdade de Medicina Veterinária, Universidade Estadual Paulista (Unesp-Araçatuba), Rua Clóvis Pestana 793, Jardim Dona Amélia, Araçatuba, SP 16050-680, Brasil. *Autor para correspondência: leydsonf@fmva.unesp.br

${ }^{3}$ Faculdade de Medicina Veterinária e Zootecnia (FAMEZ), Universidade Federal de Mato Grosso do Sul (UFMS), Av. Costa e Silva s/n, Cidade Universitária, Campo Grande, MS 79070-900, Brasil

${ }^{4}$ Pós-Graduando em Ciência Animal, Faculdade de Medicina Veterinária, Unesp-Araçatuba, Rua Clóvis Pestana 793, Jardim Dona Amélia, Araçatuba, SP 16050-680, Brasil.

${ }^{5}$ Docente do Departamento de Apoio à Produção e Saúde Animal, Faculdade de Medicina Veterinária, Unesp-Araçatuba, Rua Clóvis Pestana 793, Jardim Dona Amélia, Araçatuba, SP 16050-680, Brasil.
}

quatros grupos experimentais: PN (cordeiros nascidos de parto normal, $n=15$, média de 146 dias de gestação); PNDEX (cordeiros nascidos de parto normal, cujas mães receberam 16 mg de dexametasona aos 141 de gestação, $\mathrm{n}=8$, média de 143 dias de gestação); PRE (cordeiros prematuros nascidos de cesarianas aos 138 dias de gestação, $\mathrm{n}=10$ ) e PREDEX (cordeiros prematuros nascidos de cesarianas aos 138 dias de gestação, cujas mães receberam $16 \mathrm{mg}$ de dexametasona dois dias antes do parto, $\mathrm{n}=9$ ). Imediatamente após o nascimento, os cordeiros de todos os grupos apresentaram quadro de acidose respiratória ( $\mathrm{pH}$ baixo e $\mathrm{pCO}_{2}$ elevada), com maior ênfase nos animais prematuros. A concentração de $\mathrm{HCO}_{3}{ }^{-}$diminuiu entre 15 e 60 minutos de vida, principalmente nos grupos PRE e PREDEX, com posterior aumento no M24h. Os valores de diferença de base foram menores nos cordeiros prematuros, os quais apresentaram respiração abdominal, intensa 
dispneia e grande quantidade de líquido pulmonar. A estabilização do equilíbrio ácidobase ocorreu em todos os animais ao longo das primeiras 24 horas de vida. A dexametasona teve influência positiva sobre a condição clínica dos animais prematuros, resultando em adequada ventilação e perfusão tecidual, o que garantiu maior taxa de sobrevivência.

TERMOS DE INDEXAÇÃO: Corticoterapia, hemogasométria, cordeiros, borregos, ácidobase, prematuridade, dexametasona.

\section{INTRODUÇÃO}

O período neonatal representa estágio crítico no desenvolvimento, pois os animais recém-nascidos necessitam adaptar-se à vida extrauterina. As contrações uterinas e a ruptura das membranas fetais causam alterações na circulação feto-placentária, o que induz à acidose mista respiratória e metabólica transitória. Esta condição se reverte com o início das atividades respiratória e renal, em virtude do novo padrão de circulação sanguínea no ambiente extrauterino (Lisbôa et al. 2002).

Condições não fisiológicas, como o nascimento prematuro e a inabilidade de expansão pulmonar, resultam em quadros de asfixia precoce e tardia, sendo o desequilíbrio respiratório mais acentuado e a recuperação mais tardia (Ávila et al. 2014). Nos recém-nascidos que conseguem sobreviver há elevado risco de acidose metabólica e diminuição da capacidade de termogênese, o que leva à condição de hipotermia (Nowak et al. 2000).

A terapia com corticosteroides nas fêmeas gestantes tem sido implantada a fim de reduzir efeitos adversos em animais com nascimento precoce ou dificultoso. Dessa forma, o fármaco mimetiza a secreção fetal de cortisol, e induz à capacidade de realizar termogênese sem tremores musculares (Bispham et al. 1999). Além disso, causa alterações estruturais no parênquima pulmonar, principalmente de animais prematuros, elevando, de maneira transitória, a quantidade de surfactante (Jobe \& Ikegami 2000).

Este estudo objetivou avaliar as variáveis hemogasométricas de cordeiros nascidos a termo e prematuros ao longo das 48 horas iniciais de vida, mediante a administração materna de corticosteroide.

\section{MATERIAL E MÉTODOS}

Foram constituídos quatro grupos experimentais de ovinos mestiços da raça Suffolk, a saber: grupo PN: 15 cordeiros nascidos de partos normais (média de 146 dias de gestação); grupo PNDEX: oito cordeiros nascidos de partos normais, cujas mães receberam $16 \mathrm{mg}$ de dexametasona (Azium ${ }^{\circledR}$, Schering-Plough) , por via intramuscular, aos 141 dias de gestação (média de 143 dias); Grupo PRE: dez cordeiros prematuros, nascidos por meio de cesarianas realizadas aos 138 dias de gestação; Grupo PREDEX: nove cordeiros prematuros, nascidos por meio de cesarianas realizadas aos 138 dias de gestação, cujas mães receberam 16 mg de dexametasona, por via intramuscular, dois dias antes da cirurgia (aos 136 dias).

As ovelhas foram mantidas em piquetes com Brachiaria spp. e suplementadas com ração para ovinos fornecida uma vez ao dia, sendo colocadas em baias no período próximo ao parto (aos 141 dias de gestação para os grupos PN e PNDEX e aos 136 dias para os grupos PRE e PREDEX), com o fornecimento de silagem de milho e água à vontade.

Adotou-se um protocolo de sincronização de estro nas ovelhas dos grupos experimentais. Foram utilizadas esponjas vaginais de progesterona (Progespon ${ }^{\circledR}$, Syntex), e administração de gonadotrofina coriônica equina (eCG-Novormon ${ }^{\circledR}$, Schering-Plough) e prostaglandina (Lutalyse ${ }^{\circledR}$, Pfizer) por via intramuscular. 0 carneiro utilizado como reprodutor foi marcado na região peitoral com tinta à base de pó colorido e óleo, com tons mais claros para tons mais escuros, aplicada a cada dois dias e trocada a intervalos de 15 dias para facilitar a visualização das ovelhas cobertas, podendo assim, estimar a data da cobertura. As ovelhas foram avaliadas por exame ultrassonográfico (DP 2200 Vet, Mindray) abdominal para confirmação da gestação entre 45 e 60 dias após a última data de cobertura. Nas cesarianas realizou-se bloqueio paravertebral proximal nos ramos nervosos das vértebras T13, L1 e L2, utilizando-se cloridrato de lidocaína (Xylestesin ${ }^{\circledR} 2 \%$, Cristália), no volume de $5 \mathrm{~mL}$ em cada ponto dorsal e ventral aos processos transversos. Adicionalmente, associou-se à anestesia peridural lombossacra (L6-S1) sulfato de morfina (Dimorf ${ }^{\circledR}$, Cristália) na dose de $0,1 \mathrm{mg} / \mathrm{kg}$, diluída em $5 \mathrm{~mL}$ de solução fisiológica. 0 procedimento cirúrgico foi realizado com as ovelhas colocadas em decúbito lateral direito, para incisão em região do flanco esquerdo, conforme técnica descrita por Tibary \& Van Metre (2004).

Os cordeiros nascidos de partos normais permaneceram com as mães, ingerindo colostro à vontade. Os animais nascidos de cesarianas foram acompanhados e alimentados com colostro proveniente de banco de colostro bovino, fornecido por mamadeiras nas primeiras horas de vida.

Amostras de sangue venoso foram colhidas logo após o nascimento (M0h), aos 15 minutos (M15min), aos 60 minutos (M60min), às 24 horas (M24h) e às 48 horas de vida (M48h). Após assepsia local, realizou-se punção da veia jugular, em condições de anaerobiose, por meio de seringas plásticas descartáveis (Drihep $^{\mathrm{TM}}$ A-Line ${ }^{\mathrm{TM}}$, Becton Dickinson Company), contendo heparina lítio cálcio (80 UI de heparina) para volume de 1,6 mL, acopladas a agulhas hipodérmicas $25 \times 0,7 \mathrm{~mm}$. Quando presentes, o ar residual e as bolhas foram desprezados, e a seringa mantida selada e armazenada em recipiente térmico contendo água e gelo reciclável, sem contato direto, até o seu processamento, realizado, invariavelmente, em até 15 minutos após a colheita, como recomendado por Lisbôa et al. (2002).

Efetuou-se a determinação dos valores de $\mathrm{pH}$, pressão parcial de gás carbônico $\left(\mathrm{pCO}_{2}\right)$, bicarbonato $\left(\mathrm{HCO}_{3}^{-}\right)$e excesso/déficit de base (BE) em analisador clínico eletrônico portátil (i-Stat ${ }^{\circledR}$ Portable Clinical Analyzer, Abbott Laboratories), utilizando-se cartuchos específicos (EG7+ Cartridge, Abbott Laboratories.) de acordo com as recomendações do fabricante, sendo calibrado automaticamente antes do processamento das amostras. Os valores de $\mathrm{pH}$ e $\mathrm{pCO}_{2}$ foram ajustados pelo aparelho, de acordo com a temperatura retal de cada animal, aferida com termômetro clínico digital.

Os dados foram submetidos à análise de variância com medidas repetidas, sendo as médias comparadas pelo teste de Tukey no nível de significância de 5\%. A variável excesso/déficit de base (BE) foi analisada pelo teste de Kruskal-Wallis para comparar os grupos em cada momento e pelo teste de Friedman para comparar os momentos em cada grupo, seguido do teste de Dunn para comparações múltiplas. As análises estatísticas foram efetuadas empregando-se o programa SAS (Statistical Analysis System - SAS Institute Inc., release 9.2, Cary:NC, 2008), sendo consideradas significativas quando $\mathrm{p}<0,05$ (Zar 1998). 


\section{RESULTADOS E DISCUSSÃO}

Os resultados das análises hemogasométricas, valores médios e desvios-padrão de $\mathrm{pH}, \mathrm{pCO}_{2}, \mathrm{HCO}_{3}^{-}$e $\mathrm{BE}$ do sangue venoso dos cordeiros dos grupos PN, PNDEX, PRE e PREDEX, do nascimento as 48 horas de vida, estão descritos nos Quadros 1 e 2.

Foram avaliados 15 e oito cordeiros nos grupos PN e PNDEX, do nascimento às 48 horas de vida. Os grupos PRE e PREDEX começaram com dez e nove cordeiros, respectivamente. Contudo, em virtude da ocorrência de óbitos no decorrer do período experimental, restaram, ao final do estudo, apenas três e sete cordeiros, nos respectivos grupos (70\% e 20\% de mortalidade nos grupos PRE e PREDEX).

Imediatamente após o nascimento, os cordeiros de todos os grupos apresentaram $\mathrm{pH}$ sanguíneo abaixo dos valores considerados fisiológicos para a espécie ovina (Ortolani
2003), indicando quadro de acidose respiratória (Quadro 1). 0 parto representa estresse considerável para o feto, uma vez que as contrações uterinas afetam o fluxo sanguíneo placentário, causando hipóxia e hipercapnia (Ávila et al. 2014, Lisbôa et al. 2002). Esta condição é agravada pela reduzida capacidade pulmonar (hipoventilação) que não garante a remoção do $\mathrm{CO}_{2}$ na mesma proporção em que é produzido, causando diminuição da concentração hidrogeniônica do sangue (Ortolani 2003, Piccione et al. 2006). Os elevados valores médios de $\mathrm{pCO}_{2}$ encontrados em todos os grupos no presente estudo corroboram tais informações, principalmente logo após o nascimento e nos primeiros 15 minutos de vida (Quadro 1).

Nos animais recém-nascidos, a hipóxia e hipercapnia favorecem distribuição sanguínea desigual entre os tecidos (Piccione et al. 2006). Nos momentos que antecedem

Quadro 1. Média ( $\overline{\mathbf{x}}$ ) e desvio-padrão (SD) do potencial hidrogeniônico $(\mathrm{pH})$, pressão parcial de dióxido de carbono $\left(\mathrm{pCO}_{2}, \mathrm{mmHg}\right)$ e concentração de bicarbonato $\left(\mathrm{HCO}_{3}^{-}, \mathrm{mmol} / \mathrm{L}\right)$ no sangue venoso de cordeiros nascidos de parto normal (PN), nascidos de parto normal de mães que receberam dexametasona aos 141 dias de gestação (PNDEX), nascidos prematuramente por cesariana aos 138 dias de gestação (PRE) e nascidos prematuramente aos 138 dias de gestação de mães que receberam dexametasona 48 horas antes (PREDEX), desde o nascimento até as 48 horas de vida. Araçatuba-SP, 2015

\begin{tabular}{lccccc}
\hline Variável & Momento & $\mathrm{PN}$ & PNDEX & PRE & $\begin{array}{c}\text { PREDEX } \\
\end{array}$ \\
& & $\overline{\mathrm{x}} \pm \mathrm{S}$ & $\overline{\mathrm{x}} \pm \mathrm{S}$ & $\overline{\mathrm{x}} \pm \mathrm{S}$ & $\overline{\mathrm{x}} \pm \mathrm{S}$ \\
\hline $\mathrm{pH}$ & M0h & $7,14 \pm 0,08^{\mathrm{Abc}}$ & $7,22 \pm 0,05^{\mathrm{Ac}}$ & $7,04 \pm 0,14^{\mathrm{Bb}}$ & $7,10 \pm 0,11^{\mathrm{Bb}}$ \\
& M15min & $7,17 \pm 0,07^{\mathrm{ABbc}}$ & $7,24 \pm 0,05^{\mathrm{Abc}}$ & $6,94 \pm 0,15^{\mathrm{Cb}}$ & $7,08 \pm 0,12^{\mathrm{Bb}}$ \\
& M60min & $7,22 \pm 0,09^{\mathrm{ABb}}$ & $7,28 \pm 0,06^{\mathrm{Ab}}$ & $7,05 \pm 0,14^{\mathrm{Cb}}$ & $7,14 \pm 0,09^{\mathrm{BCb}}$ \\
& M24h & $7,38 \pm 0,05^{\mathrm{Aba}}$ & $7,45 \pm 0,03^{\mathrm{Aa}}$ & $7,37 \pm 0,03^{\mathrm{Ba}}$ & $7,35 \pm 0,04^{\mathrm{Ba}}$ \\
& M48h & $7,41 \pm 0,05^{\mathrm{a}}$ & $7,40 \pm 0,05^{\mathrm{a}}$ & $7,39 \pm 0,02^{\mathrm{a}}$ & $7,34 \pm 0,06^{\mathrm{a}}$ \\
$\mathrm{pCO}_{2}(\mathrm{mmHg})$ & M0h & $72,3 \pm 7,1^{\mathrm{Ba}}$ & $72,2 \pm 7,2^{\mathrm{Ba}}$ & $93,0 \pm 16,8^{\mathrm{Aa}}$ & $82,8 \pm 18,1^{\mathrm{ABa}}$ \\
& M15min & $70,2 \pm 8,3^{\mathrm{Ba}}$ & $66,5 \pm 8,5^{\mathrm{Ba}}$ & $98,1 \pm 28,4^{\mathrm{Aa}}$ & $75,0 \pm 14,8^{\mathrm{Bb}}$ \\
& M60min & $64,4 \pm 10,6^{\mathrm{Bb}}$ & $63,9 \pm 5,2^{\mathrm{Ba}}$ & $80,3 \pm 19,3^{\mathrm{Aab}}$ & $69,5 \pm 6,9^{\mathrm{ABab}}$ \\
& M24h & $50,4 \pm 4,1^{\mathrm{Abc}}$ & $45,2 \pm 3,4^{\mathrm{Bb}}$ & $52,4 \pm 1,7^{\mathrm{ABb}}$ & $56,6 \pm 6,9^{\mathrm{Ab}}$ \\
$\mathrm{HCO3}^{-}(\mathrm{mmol} / \mathrm{L})$ & M48h & $50,1 \pm 6,2^{\mathrm{c}}$ & $47,2 \pm 9,2^{\mathrm{b}}$ & $57,3 \pm 5,8^{\mathrm{b}}$ & $56,0 \pm 9,1^{\mathrm{b}}$ \\
& M0h & $23,9 \pm 3,8^{\mathrm{Bb}}$ & $28,1 \pm 2,3^{\mathrm{a}}$ & $24,0 \pm 3,2^{\mathrm{Bab}}$ & $24,6 \pm 1,5^{\mathrm{ABab}}$ \\
& M15min & $24,9 \pm 4,5^{\mathrm{ABb}}$ & $27,8 \pm 3,3^{\mathrm{a}}$ & $20,5 \pm 4,0^{\mathrm{Bb}}$ & $21,9 \pm 2,5^{\mathrm{ABb}}$ \\
& M60min & $26,2 \pm 5,7^{\mathrm{ABb}}$ & $28,8 \pm 2,0^{\mathrm{A}}$ & $22,3 \pm 3,1^{\mathrm{Bab}}$ & $22,9 \pm 3,1^{\mathrm{ABb}}$ \\
& M24h & $29,5 \pm 3,2^{\mathrm{a}}$ & $30,3 \pm 3,6$ & $29,6 \pm 2,5^{\mathrm{ab}}$ & $30,1 \pm 2,2^{\mathrm{a}}$ \\
& M48h & $31,1 \pm 3,7^{\mathrm{a}}$ & $28,0 \pm 4,9$ & $34,1 \pm 1,5^{\mathrm{a}}$ & $29,0 \pm 3,5^{\mathrm{a}}$
\end{tabular}

Médias seguidas de letras diferentes, maiúscula na linha e minúscula na coluna, diferem entre si pelo teste de Tukey $(\mathrm{p}<0,05)$. A ausência de letras implica que não há diferença estatística.

Quadro 2. Mediana (Md), mínimo (Mín) e máximo (Máx) do excesso/déficit de base (BE, mmol/L) no sangue venoso de cordeiros nascidos de parto normal (PN), nascidos de parto normal de mães que receberam dexametasona aos

141 dias de gestação (PNDEX), nascidos prematuramente por cesariana aos 138 dias de gestação (PRE) e nascidos prematuramente aos 138 dias de gestação de mães que receberam dexametasona, 48 horas antes (PREDEX), desde o nascimento até as 48 horas de vida. Araçatuba-SP, 2015

\begin{tabular}{|c|c|c|c|c|c|c|c|c|}
\hline \multirow[t]{3}{*}{ Momento } & \multicolumn{7}{|c|}{$\mathrm{BE}(\mathrm{mmol} / \mathrm{L})$} & \\
\hline & \multicolumn{2}{|l|}{ PN } & \multicolumn{2}{|l|}{ PNDEX } & \multirow{2}{*}{$\begin{array}{c}\text { PRE } \\
\text { Md }\end{array}$} & \multicolumn{3}{|c|}{ PREDEX } \\
\hline & Md & Mín-Max & Md & Mín-Max & & Mín-Max & Md & Mín-Max \\
\hline $\mathrm{MOh}$ & $-3,0^{A b c}$ & -18 & $0,3^{\mathrm{Ab}}$ & -8 & $-7,0^{\text {Bab }}$ & -17 & $-6,0^{\mathrm{Bab}}$ & $-9,5$ \\
\hline M15min & $-2,0^{\mathrm{ABbc}}$ & -19 & $1,2^{\mathrm{Ab}}$ & -10 & $-12,0^{\mathrm{Cb}}$ & -16 & $-9,0^{\text {BCab }}$ & -10 \\
\hline M60min & $0,0^{\mathrm{ABab}}$ & -17 & $3,0^{\text {Aab }}$ & $-8,7$ & $-11,0^{\text {Bab }}$ & -11 & $-6,5^{\mathrm{Bab}}$ & -14 \\
\hline M24h & $6,0^{\mathrm{a}}$ & -12 & $6,0^{\mathrm{a}}$ & $2,2-14,0$ & $6,0^{\mathrm{ab}}$ & $1,0-7,0$ & $5,3^{\mathrm{a}}$ & $1,5-8,0$ \\
\hline M48h & $5,0^{\mathrm{a}}$ & $1,0-11,0$ & $2,1^{\mathrm{ab}}$ & $-14,3$ & $9,0^{\mathrm{a}}$ & $8,0-11,0$ & $2,0^{\mathrm{a}}$ & $-9,7$ \\
\hline
\end{tabular}

Medianas seguidas de letras diferentes, maiúscula na linha e minúscula na coluna, diferem entre si pelo teste de Dunn $(\mathrm{p}<0,05)$. A ausência de letras implica que não há diferença estatística. 
o nascimento, o feto é obrigado a suportar uma condição de hipoxemia em virtude do prejuízo nas trocas gasosas materno-fetais. A maior taxa de metabolismo anaeróbico com a geração do ácido lático é, portanto, o resultado final deste evento (Lisbôa et al. 2002).

$\mathrm{Na}$ primeira hora de vida as alterações encontradas nas variáveis $\mathrm{pH}$ e $\mathrm{pCO}_{2}$ em todos os grupos foram evidentes, destacando-se os grupos de animais prematuros, que apresentaram os menores valores médios de $\mathrm{pH}$ e maiores valores médios de $\mathrm{pCO}_{2}$ aos 15 minutos de vida (Quadro 1). Entretanto, houve diferença nestas variáveis entre os grupos PRE e PREDEX aos 15 minutos de vida, o que evidencia a influência da dexametasona na condição clínica dos animais prematuros. Verificou-se melhora da capacidade respiratória e início mais rápido da ventilação, embora, ainda, irregular e superficial, em relação ao grupo PRE, no período crítico para a sobrevivência dos prematuros, compreendido entre o nascimento e os primeiros 15 minutos de vida.

Ao longo do período de 48 horas de avaliação, houve normalização do $\mathrm{pH}$ e redução da $\mathrm{pCO}_{2}$ tanto nos cordeiros nascidos em tempo gestacional fisiológico para a espécie por meio de parto normal, quanto nos prematuros nascidos por cesariana, aos 138 dias de gestação. Isso ocorreu provavelmente pela resposta compensatória, com o incremento da ventilação e o tamponamento químico (Carlson \& Bruss 2008). $\mathrm{O}$ aumento na concentração de $\mathrm{HCO}_{3}{ }^{-}$por elevação na retenção renal deste íon, entretanto, raramente é responsável pela correção do $\mathrm{pH}$ durante as primeiras horas de vida, uma vez que este mecanismo requer alguns dias para alcançar sua plena eficácia (Piccione et al. 2006).

A concentração de $\mathrm{HCO}_{3}{ }^{-}$diminuiu entre 15 e 60 minutos de vida nos grupos PRE e PREDEX (Quadro 1), embora os valores estejam dentro do intervalo considerado fisiológico para a espécie (Ortolani, 2003). Contudo, tanto nos grupos de animais prematuros como nos nascidos a termo houve aumento na concentração de $\mathrm{HCO}_{3}^{-}$no $\mathrm{M} 24 \mathrm{~h}$, indicando a estabilização do quadro metabólico nestes animais.

Quanto ao excesso/déficit de base (BE), os cordeiros prematuros apresentaram valores mais baixos do nascimento até 60 minutos de vida (Quadro 2), concordando com Ravary-Plumioen (2009), que citou que, em casos de distocia ou hipóxia, o $\mathrm{pH}$ pode diminuir a valores iguais ou inferiores a 7,0 e o BE variar de -10 a $-15 \mathrm{mmol} / \mathrm{L}$. Os cordeiros nascidos de parto normal apresentaram progressiva elevação do BE neste período, contrariando Camargo (2010) e Bovino (2011), que verificaram diminuição do BE nos animais de parto normal durante os primeiros $15 \mathrm{mi}$ nutos de vida. No presente estudo, os cordeiros prematuros apresentaram elevação de BE às 24 e às 48 horas, denotando estabilização do quadro metabólico; esses resultados contrariam Rodrigues et al. (2007), porém são coincidentes com os dados de Camargo (2010) e Bovino (2011), indicando que o período de 24 horas foi adequado para a completa recuperação do equilíbrio ácidobase.

No presente estudo, os cordeiros prematuros apresentaram dificuldade para iniciar os movimentos respiratórios, resultando em atraso no início da ventilação pulmonar. Os cordeiros cujas mães receberam dexametasona (PREDEX) tiveram mais facilidade neste processo, além de menor quantidade de líquido presente nas narinas e traqueia. Logo após o rompimento do cordão umbilical, a diminuição da tensão de oxigênio e o aumento da concentração de dióxido de carbono estimulam os reflexos respiratórios, com subsequente expansão dos pulmões (Nagy 2009). Na presença de distocia prolongada e/ou em situações em que a hipercapnia ou acidose seja iniciada antes da expulsão do feto, a depressão do sistema nervoso pode ser severa o suficiente para prejudicar os reflexos que iniciam a respiração (Camargo 2010, Bovino 2011).

A remoção de líquido do sistema pulmonar é crítica e essencial para que a ventilação normal e a oxigenação sanguínea ocorram (Nagy 2009). Uma parte deste líquido é eliminada do organismo durante o parto; entretanto, a maior parte é rapidamente absorvida através das paredes alveolares para dentro do interstício no início da respiração (Egan et al. 1975). Neste estudo, os resultados hemogasométricos e a observação clínica mostraram que os animais prematuros foram menos eficientes na remoção do líquido pulmonar e na inicialização do processo respiratório. Todavia, os cordeiros sob a influência da dexametasona tiveram mais facilidade para respirar, com menor quantidade de líquido aspirado das vias aéreas superiores.

Os cordeiros prematuros (PRE) apresentaram do nascimento aos 60 minutos de vida respiração predominantemente abdominal, associada à intensa dispneia, com movimentos irregulares muitas vezes interrompidos por episódios de apneia. À auscultação pulmonar, foi possível constatar presença de respiração ruidosa, áspera, caracterizada por crepitação grossa, indicando grande quantidade de líquido intrapulmonar. Os cordeiros do grupo PREDEX, embora também apresentassem respiração predominantemente abdominal, com padrão irregular e denotando grande quantidade de líquido nos pulmões, não apresentaram episódios de apneia, conseguindo manter a continuidade dos movimentos respiratórios. Isso pode ser explicado pelo efeito benéfico da dexametasona sobre a maturação estrutural pulmonar, culminando em melhor capacidade de expansão alveolar (Jobe \& Ikegami 2000, Jobe 2001, Moss 2006).

Benesi (1993) encontrou distúrbios respiratórios semelhantes em bezerros acometidos por asfixia neonatal, caracterizados por apresentação de tipo respiratório predominantemente abdominal e associação de ruídos evidenciados como crepitação grossa. As descrições desse autor e do presente estudo correlacionam-se aos citados em casos de evidente imaturidade fetal, identificados pelas alterações dos padrões fisiológicos dos achados de hemogasometria (Bleul 2009).

\section{CONCLUSÃO}

Os cordeiros prematuros, nascidos por cesariana aos 138 dias de gestação, apresentam acidose respiratória e metabólica mais intensa que os cordeiros nascidos por parto normal em tempo gestacional fisiológico para a espécie; a estabilização do equilíbrio ácidobase ocorre ao longo das primeiras 24 horas de vida, com a normalização das variáveis hemogasométricas; a dexametasona tem influência positiva sobre a condição clínica dos animais prematuros, 
resultando no início mais precoce dos movimentos respiratórios e, consequentemente, em maior chance de sobrevivência.

Agradecimentos.- Os autores agradecem o aporte financeiro da Fundação de Amparo à Pesquisa do Estado de São Paulo (FAPESP) para o desenvolvimento do projeto (Processo 2010/19497-4) e à concessão de bolsa de doutorado (Processo 2011/01123-3).

\section{REFERÊNCIAS}

Ávila L G., Bovino F., Camargo D.G., Souza N.C., Santos G.G.F., Deschk M., Mendes L.C.N. \& Feitosa F.L.F. 2014 Aplicação materna de glicocorticoide nos parâmetros vitais de cordeiros nascidos a termo e prematuros. Ciência Rural 44(6):1106-1112.

Benesi FJ. 1993. Síndrome asfixia neonatal dos bezerros. Importância e avaliação crítica. Arq. Esc. Med. Vet. Univ. Fed. Bahia 16:38-48.

Bispham J., Heasman L., Clarke L., Ingleton P.M., Stephenson T. \& Symonds M.E. 1999. Effect of maternal dexamethasone treatment and ambient temperature on prolactin receptor abundance in brown adipose and hepatic tissue in the fetus and newborn lamb. J. Neuroendocrinol. 11(11):849-856

Bleul U. 2009. Respiratory distress syndrome in calves. Vet. Clin. Food Anim, 25(1):179-193

Bovino F. 2011. Determinação do escore Apgar, dos valores hemogasométricos e do proteinograma sérico em cordeiros (Ovis aries) nascidos de partos normais e de cesarianas. Dissertação de Mestrado em Ciência Animal, Faculdade de Medicina Veterinária de Araçatuba, Universidade Estadual Paulista, Araçatuba. 90p.

Camargo D.G. 2010. Avaliação do sistema APGAR (modificado por Born 1981) e dos níveis de cortisolemia, glicemia e de gases sanguíneos em cabritos nascidos de partos eutócicos e de cesariana. Dissertação de Mestrado em Ciência Animal, Faculdade de Medicina Veterinária de Araçatuba, Universidade Estadual Paulista, Araçatuba. 93p.

Carlson G.P. \& Bruss M. 2008. Fluid, electrolyte, and acid-base balance. In: Kaneko J.J. (Ed.), Clinical Biochemistry of Domestic Animal. $6^{\underline{a}}$ ed. Academic, San Diego. 559p.
Egan E.A., Olver R.E. \& Strang L.B. 1975. Changes in non-electrolyte permeability of alveoli and the absorption of lung liquid at the start of breathing in the lamb. J. Physiol. 244(1):161-179. Disponível em <http://www.ncbi.nlm.nih.gov/pmc/articles/PMC1309098/pdf/jphysiol00838-0160.pdf> Acesso em 3 fev. 2016.

Jobe A.H. \& Ikegami M. 2000. Lung development and function in preterm infants in the surfactante treatment era. Annu Rev. Physiol. 62:825-846

Jobe A.H. 2001. Glucocorticoids, inflammation and the perinatal lung. Semin. Neonatol. 6(4):331-342.

Lisbôa J.A.N., Benesi F.J., Leal M.L.R. \& Teixeira C.M.C. 2002. Efeito da idade sobre o equilíbrio ácido básico de bezerras sadias no primeiro mês de vida. Braz. J. Vet. Res. Anim. Sci. 39(3):136-142.

Moss T.J.M. 2006. Respiratory Consequences of Preterm Birth. Clin. Experiment. Pharmacol. Physiol. 33(3):280-284.

Nagy D.W. 2009. Resuscitation and critical care of neonatal calves. Vet. Clin. Food Anim. 25(1):1-11.

Nowak R., Porter R.H., Lévy F., Orgeur P. \& Schaal B. 2000. Role of mother-young interactions in the survival of offspring in domestic mammals. Rev Reprod, 5(3):153-63.

Ortolani E.L. 2003. Diagnóstico e tratamento de alterações ácido-básicas em ruminantes. Anais do Primeiro Simpósio de Patologia Clínica Veterinária da Região Sul do Brasil, Porto Alegre, RS.

Piccione G., Costa A., Bertolucci C., Borruso M., Pennisi P. \& Caola G. 2006. Acid-base balance modifications in the lamb and goat kids during the first week of life. Small Rumin. Res. 63(3):304-308.

Ravary-Plumioen B. 2009. Resuscitation procedures and life support of the newborn calf. Revue Méd. Vét. 160(8-9):410-419.

Rodrigues J.A., Veiga G.A.L., Silva L.C., Lúcio C.F. \& Vannuchi C.I. 2007. Avaliação por escore APGAR, temperatura e hemogasometria arterial em neonatos da espécie ovina. Anais do Congresso Brasileiro de Reprodução Animal, Curitiba, PR.

Tibary A. \& Van Metre D. 2004. Surgery of the sheep and goat reproductive system and urinary tract, p 527-547. In: Fubini S.L. \& Ducharme N.G. (Eds), Farm Animal Surgery. W.B. Saunders, St Louis. 547p.

Zar J.H. 1998. Biostatistical Analysis. 4⿳亠丷a ed. Prentice-Hall, New Jersey. 930p. 